\title{
Apparent Detection via New Telescopes with Concave Lenses of Otherwise Invisible Terrestrial Entities (ITE)
}

\author{
Ruggero Maria Santilli \\ Thunder Energies Corporation, Florida, U. S. A.
}

Email address:

research@thunder-energies.com

To cite this article:

Ruggero Maria Santilli. Apparent Detection via New Telescopes with Concave Lenses of Otherwise Invisible Terrestrial Entities (ITE). American Journal of Modern Physics. Vol. 5, No. 3, 2016, pp. 45-53. doi: 10.11648/j.ajmp.20160503.14

Received: December 24, 2015; Accepted: December 25, 2015; Published: June 8, 2016

\begin{abstract}
By using telescopes with concave lenses, known as Santilli telescopes (trademark and patent pending by the U.S. publicly traded company Thunder Energies Corporation), we review preceding evidence for the apparent existence of antimatter galaxies, antimatter asteroids and antimatter cosmic rays. Independently from these astrophysical detections, we present for the first time evidence for the apparent existence of entities in our terrestrial environment that are solely visible via telescopes with concave lenses, while being invisible to our eyes and to conventional Galileo telescopes with convex lenses, which entities leave dark images in the background of digital cameras attached to the Santilli telescopes. These entities are here called Invisible Terrestrial Entities of the first kind (ITE-1) or dark ITE. We then present, also for the first time, evidence for the apparent existence in our terrestrial environment of additional entities that are also visible to telescopes with concave lenses while being invisible to our eyes and to conventional telescopes with convex lenses, which entities leave bright images in the background of digital cameras. These additional entities are here called Invisible Terrestrial Entities of the second kind (ITE-2) or bright ITE. It is pointed out that both types of entities generally move in the night sky over sensitive areas, and their behavior generally suggests unauthorized surveillance. This paper has been motivated by the significance and diversification of the collected evidence, as well as available independent confirmations, that warrant systematic inspections of the sky over our sensitive civilian, industrial, and military installations via telescopes with concave lenses, so as to detect possible unauthorized surveillance.
\end{abstract}

Keywords: Antimatter, Santilli Telescope, Invisible Terrestrial Entities

\section{Introduction}

As it is well established in particle physics laboratories, matter and antimatter particles "annihilate" at mutual contact by transforming their masses into light. One of the necessary conditions for a consistent, quantitative representation of this experimental evidence is that all characteristics of antimatter must be opposite to those of matter.

It as also been established that the use of 20th century mathematics for the representation of both, matter and antimatter, leads to predictable catastrophic inconsistencies.

Therefore, a consistent, quantitative representation of matter-antimatter annihilation requires the continued use of conventional mathematics for the representation of matter, while antimatter must be represented with a basically new mathematics characterized by a suitable conjugation of 20th century mathematics known as the isodual map (technically given by an anti-Hermitean map).

In summer 1993, while visiting the Joint Institute for Nuclear Research in Dubna, Russia, the author initiated the construction of the new mathematics needed for antimatter, with the proposal of new numbers, today known as isodual numbers, whose basic unit has the negative value -1 , thus assuring that all quantities measured with the new numbers are opposite those of matter [1].

By noting that the possible existence of antimatter galaxies must be studied at the macroscopic, and therefore classical level, thus preventing the use of quantum mechanics, and that galaxies must be assumed as being neutral, thus preventing the use of the charge for the conjugation from matter to antimatter, in the following paper [2] (also written in Dubna), the author presented the first, and, apparently, the only known classical representation of neutral antimatter based on the new isodual numbers and ensuing new mathematics. 
In summer 1995, while conducting research at the Institute for Basic Research at the Castle Prince Pignatelli in Italy, the author conducted systematic studies on the novel isodual mathematics via a step-by-step isodual image of 20th century mathematics, thus including the isodual image of functional analysis, differential calculus, algebras, geometries, etc. [3].

In spring 1996, the author presented at the First International Workshop on Anti-matter in Sepino, Italy, the prediction of isodual mathematics that light emitted by antimatter-stars, here called "antimatter-light," is different than our ordinary matter-light in an experimentally verifiable way [4].

The above prediction was based on the fact that ordinary light has no charge. Therefore, the only known consistent way to conjugate light from matter to antimatter is the map under isoduality of all other physical characteristics of light. This lead to the prediction that antimatter light has negative energy, by therefore confirming the original 1928 conception of antimatter by P. A. M. Dirac as having negative energy, this time, with the resolution of its historical inconsistencies permitted by the novel isodual mathematics.

A systematic study of the ensuing isodual theory of antimatter was presented in monograph [5] in 2006, including the isoduality of Newtonian mechanics, Galileo relativity, Einstein special and general relativity, and quantum mechanics, with the first known classical representation of the gravitational field of antimatter bodies.

In particular, Ref. [5] presented the proof that the isodual theory of antimatter verifies all known experimental data on antimatter at both the classical and particle levels. At the classical level, experimental data are verified by the interplay between the conventional Newton's equation for particle and their isodual for antiparticles, while at the particle level experimental data on antimatter are verified because the isodual map is equivalent to charge conjugation.

In Ref. [5], the author also presented the prediction at all levels of study, including the isodual Newtonian mechanics, isodual special and general relativity and isodual quantum mechanics, that matter and antimatter experience gravitational repulsion, thus including the prediction that antimatter-light is repelled by a matter gravitational field (Figure 1).

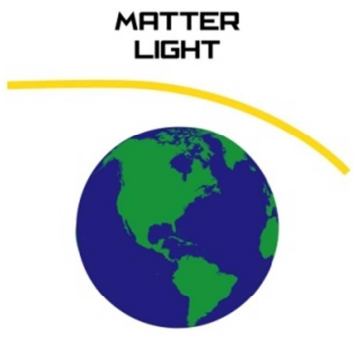

MPTTER

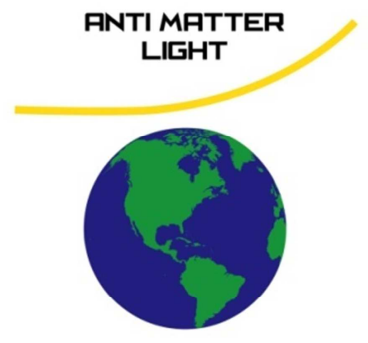

MATTER

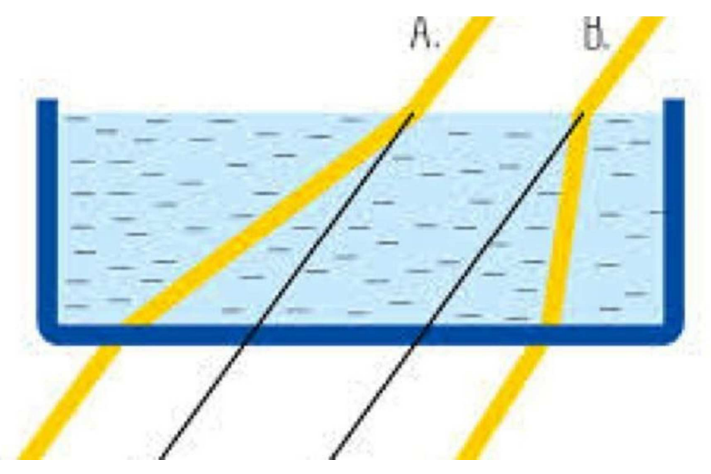

Figure 1. A necessary condition for a consistent, quantitative representation of matter-antimatter annihilation into light at contact is that "all" characteristics of antimatter are opposite those of matter. This basic requirement implies the prediction that light emitted by antimatter, viz., antimatter-light, is repelled by a matter gravitational field, and the consequential prediction that, when propagation within a matter-medium such as water, antimatter-light has a "negative" index of refraction opposite to the conventional "positive" index of refraction of matter-light.

In 2012, the author presented at the International Conference of Numerical Analysis and Applied Mathematics held in Kos, Greece, the prediction of the isodual theory of antimatter according to which, when traversing a transparent matter-medium, antimatter-light has a "negative" index of refraction (in the sense of being opposite to the conventional "positive" index of refraction of matter-light), thus requiring "concave" lenses for the focusing of it.

\section{Galileo}

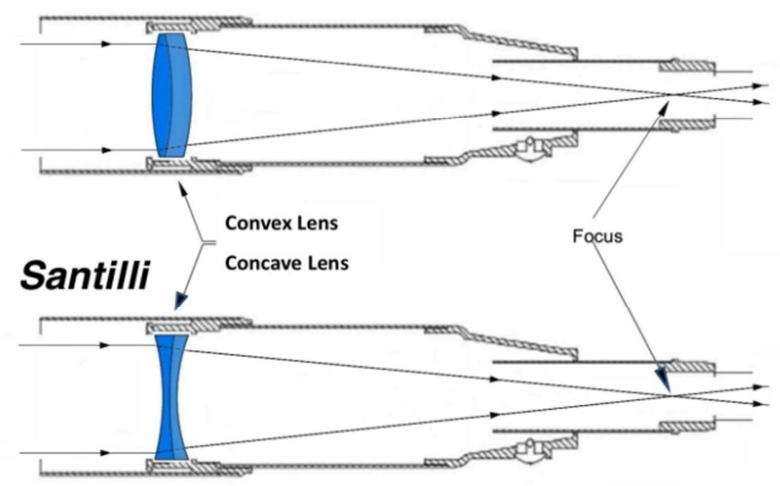

Figure 2. The first view depicts the structure of all existing, refractive, Galileo telescopes whose primary lens must be "convex" due to the positive index of refraction of matter-light. The second view depicts the structure of the novel, refractive, Santilli telescopes whose primary lens must be "concave" under the prediction that antimatter-light has a negative index of refraction (See Figure 1). The main principle of detection of the Galileo and Santilli telescopes is the following. In the Galileo telescope, all antimatterlight is dispersed by the convex lens into the internal walls of the telescope, thus permitting images of matter-light to be focused in a camera without significant interferences by antimatter-light. By contrast, ordinary matterlight is dispersed by the convex lens of the Santilli telescope, thus permitting images of antimatter-light to be focused in a camera without major interferences from matter-light.

The images shown in Figures 1 and 2 are referenced in [6].

Subsequently, the author constructed the first known telescopes with concave lenses, today known as Santilli telescopes (trademark and patent pending by the U. S. 
publicly traded company Thunder Energies Corporation, www.thunder-energies.con). Since no conventional image can be seen with concave lenses, Santilli telescopes were paired to conventional, refractive Galileo telescopes of the same size, with curvature of the primary lenses and focal distance conjugated into negative values (Figures 2 and 3).

Experimental paper [7] of 2014 presented the first known evidence of the apparent existence in our universe of antimatter galaxies, antimatter asteroids and antimatter cosmic rays via the use of a pair of $100 \mathrm{~mm}$ Galileo and Santilli telescopes with attached digital camera Cannon 600D used at ISO 1600 under a 15 seconds exposure. These first detections were independently confirmed in Refs. [8, 9].

The detections consist of black streaks over a conventional background of a digital camera attached to the Santilli telescope, which black streaks are absent in the Galileo

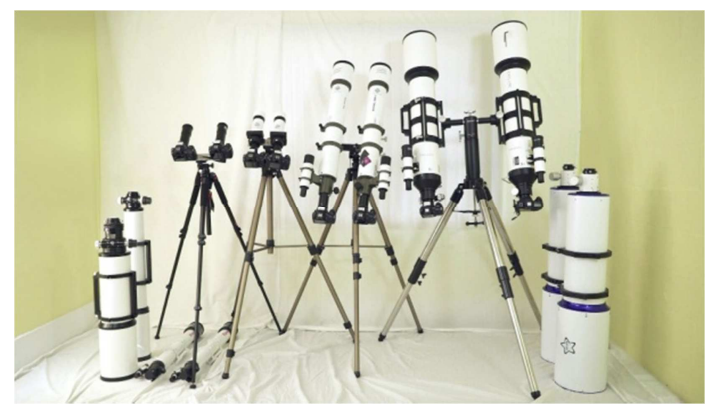

Figure 3. A view of the $50 \mathrm{~mm}, 70 \mathrm{~mm}, 100 \mathrm{~mm}, 150 \mathrm{~mm}$ and $200 \mathrm{~mm}$ pairs of Galileo and Santilli telescopes used for the detections presented in this paper, that are under production and subsequent sale by the U. S. publicly traded company Thunder Energies Corporation (www.thunderenergies.com). Santilli telescopes cannot be efficiently used alone because the human eye has a convex cornea, thus being unable to focus images of antimatter-light. The efficient use Santilli telescopes is that of pairing them with optically aligned, Galileo telescopes of the same size with exactly the same, yet opposite curvatures of the primary lenses and focal distances (Fig. 2). The Galileo telescope is then used for focusing images of matter-light. The related settings are then transferred to the Santilli telescope. Images in the Santilli telescope are considered for analysis if and only if they do not appear in the Galileo telescope, are not caused by impurities in the lens and verify other conditions. Note that the pairs of telescopes depicted in this figure are equipped with two identical cameras, one per telescope, however, detections selected for publication are generally achieved via one single camera, first used in the Galileo telescope to verify focusing, and then transferred to the Santilli telescope.

The very focusing of images via a telescope with concave lenses constitutes direct experimental evidence for the existence in nature of light with a negative index of refraction, while the black character of the streaks constitutes direct experimental evidence supporting the hypothesis of Ref.[4] according to which antimatter-light carries negative energy. In turn, both these features are considered as experimental evidence for the gravitational repulsion between matter and antimatter due to its need to achieve a negative index of refraction.

A number of independent studies exist on isodual mathematics and on the isodual theory of antimatter among which we quote: Ref. [10] on the need to conduct systematic studies on the still unknown means to detect antimatter asteroids prior to their impact on Earth that have occurred in the past without advance detection; Ref. [11] on quantitative calculation of the trajectories and speed under which antimatter asteroids can hit Earth despite the indicated gravitational repulsion from Earth; and Ref. [12] providing, a list of all known contributions in the field published in refereed journals as of early 2015, including the link to numerous PRWeb Releases on the ongoing search for antimatter galaxies in the universe.

In a language accessible to the general audience, thus without any equation, in this paper we present for the first time the detection of entities existing in our terrestrial environment here defined as including our atmosphere, terrestrial and lunar orbits and under the sea, which entities are not visible by our naked eye or via existing optical means, yet they are fully detected in cameras attached to the novel telescopes with concave lenses.

To prevent a prohibitive length as well as unnecessary repetitions, a knowledge of papers [7-9] is essential for the understanding of this paper.

\section{Detection of Invisible Terrestrial Entities of the First and Second Kind (ITE-1 and ITE-2)}

On September 5, 2015, at $9.30 \mathrm{pm}$ the author aimed a pair of $100 \mathrm{~mm}$ Galileo and Santilli telescopes at the night sky over Tampa Bay, Florida, as seen from the NE orientation of the terrace of room 775 of the Vinoy Renaissance Hotel in St. Petersburg. Both telescopes were equipped with a Sony camera model SLT-A58K set at ISO automatic and 15 seconds exposure, the two cameras being activated jointly by remote shutters.

The tests were merely intended for the search of antimatter galaxies and, consequently, the paired telescopes were aimed at the sky, but sudden clouds halted the tests and the author oriented the pair of telescopes horizontally over Tampa Bay.

To his great surprise, unidentified yet clearly visible entities immediately appeared in the screen of the camera attached to the Santilli telescope, without any enlargement, without the same entities being visible to the naked eyes, and without any corresponding image existing in the screen of the camera attached to the Galileo telescope.

This unexpected discovery triggered a novel systematic use of the pair of Galileo and Santilli telescopes, this time, for the search of entities, here called Invisible Terrestrial Entities, that are invisible to our eyes as well as to our optical instruments with convex lenses, but are otherwise fully visible via the Santilli telescope with concave lenses, and are located in our terrestrial environment, as defined in Section 1.

Following systematic tests, the author has detected the existence of at least two different types of ITE, with the acknowledgement that additional types may be identified in the future. Thanks to independent confirmations on the existence of ITE, such as that by K. Brinkman [13] and others, the author first presented the discovery of ITE on 
September 25, 2015, at an invited lecture delivered to the St. Petersburg Astronomy Club [14].

In Figures 4, 5, and 6, we report representative examples of rather numerous detections of Invisible Terrestrial Entities of the first kind (ITE-1), here defined as entities that:

1) are not visible to the human eye or to conventional optical instruments with convex lenses, but are otherwise fully visible via Santilli telescopes with concave lenses;

2) exist in our terrestrial environment as defined in Section 1 , rather than in deep astro-physical spaces; and

3) leave "dark images" in the background of digital cameras attached to Santilli telescopes.

In Figures 7 to 11, the author presents representative examples of Invisible Terrestrial Entities of the second kind (ITE-2), here defined as entities that:

1) are not visible to the human eye or to conventional optical instruments wish convex lenses, but are otherwise fully visible via Santilli telescopes with concave lenses;

2) exist in our terrestrial environment as defined in Section 1 , rather than in deep astro-physical spaces; and

3) leave "bright images" in the background of digital cameras attached to Santilli telescopes.

A first significant aspect of the detections presented in Figures 4 to 11 is that they establish beyond "credible doubt" the capability of telescopes with concave lenses to focus images visible in the background of a digital camera attached to Santilli telescopes. These images are at times, visible without enlargement, thus establishing the utilities of telescopes with concave lenses.

A second salient aspect is that the focusing of images via concave lenses is solely possible for light having a negative index of refraction in the sense of being opposite that of ordinary light (Section 1).

A third salient aspect is that the emission of antimatterlight does not mean that the entity is made up of antimatter, because the existence of the entities within our atmosphere would imply a cataclysmic explosion due to matterantimatter annihilation.

Yet another salient aspect is that the emission of antimatter-light could well be evidence that their propulsion system is based on the extraction of antimatter from space intended as a universal substratum of the entire universe with an extremely high energy density (also known as zero point energy) [15].

In short, it appears that ITE-1 consist of matter-entities in our terrestrial environment achieving locomotion via the acquisition of antimatter in their interior with consequential use of matter-antimatter propulsion, and achieve invisibility via the emission of antimatter-light as a sort of exhaust. A Post Ph.D. mathematical study of the new type of locomotion via the use of the new isodual Minkowskian geometry for antimatter is available in monograph [5].

A third salient aspect is that the creation of "dark images" in the background of digital cameras for ITE-1 (Figures 4-6) establishes that the light originating from the image has negative energy, since only a negative energy can annul the conventional positive energy existing in the pixels of the cameras. Consequently, the dark images of ITE-1 are caused by a type of light either equivalent or identical to antimatterlight as presented in the preceding section.

By contrast, ITE-2 (Figures 7 to 11) appear to be structures composed of matter which ordinary light (because of their bright character), yet possessing means to achieve invisibility via the conversion of their index of refraction from a positive to a negative value.

It should be indicated that ITE-1 appear to be located mostly in the areas of terrestrial or lunar orbits. By contrast, ITE-2 are generally located directly over sensitive civilian, industrial and military installations, and appear to behave in a way strongly suggesting their unauthorized surveillance.
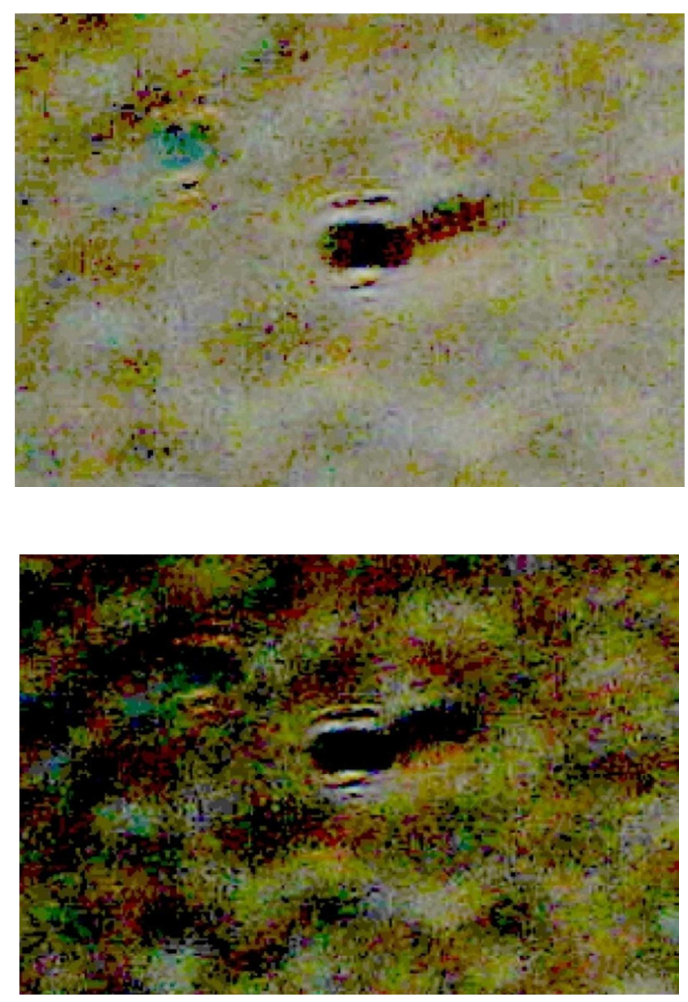

Figure 4. A view of an Invisible Terrestrial Entity of the first kind (ITE-1) detected in two different photos on September 5, 2015, in the evening sky over Tampa Bay. Florida, via the pair of $100 \mathrm{~mm}$ Galileo and Santilli telescopes with Sony Camera SLT-A58K set at ISO automatic and 15 seconds exposure. The entity is classified as an ISE-1 because it is solely detected via the Santilli telescope (thus emitting light with negative index of refraction), and it leaves a black image in the background of the digital camera (thus emitting light with negative energy). Since we are dealing with two different photos each taken with 15 seconds exposure, the entity moves at a relative small speed. Note the ridges of ordinary light surrounding the entity, which can only be explained quantitatively via the gravitational repulsion of ordinary light by the entity because, in the absence of such repulsion, ordinary light should merely experience diffraction. Note additionally that the entity "cannot" be composed of antimatter because, being within our atmosphere, it would annihilate with a cataclysmic explosion.

Therefore, the entity is predicted to be made up of ordinary matter, although emitting antimatter light. These conditions support the hypothesis of locomotion based on the extraction 
of negative energy from space conceived as a universal substratum (also known as negative point energy). According to this interpretation, the entity achieves invisibility to our eyes as well as to all conventional refractive telescopes by merely emitting antimatter-light as a kind of "exhaust" from its locomotion [15].
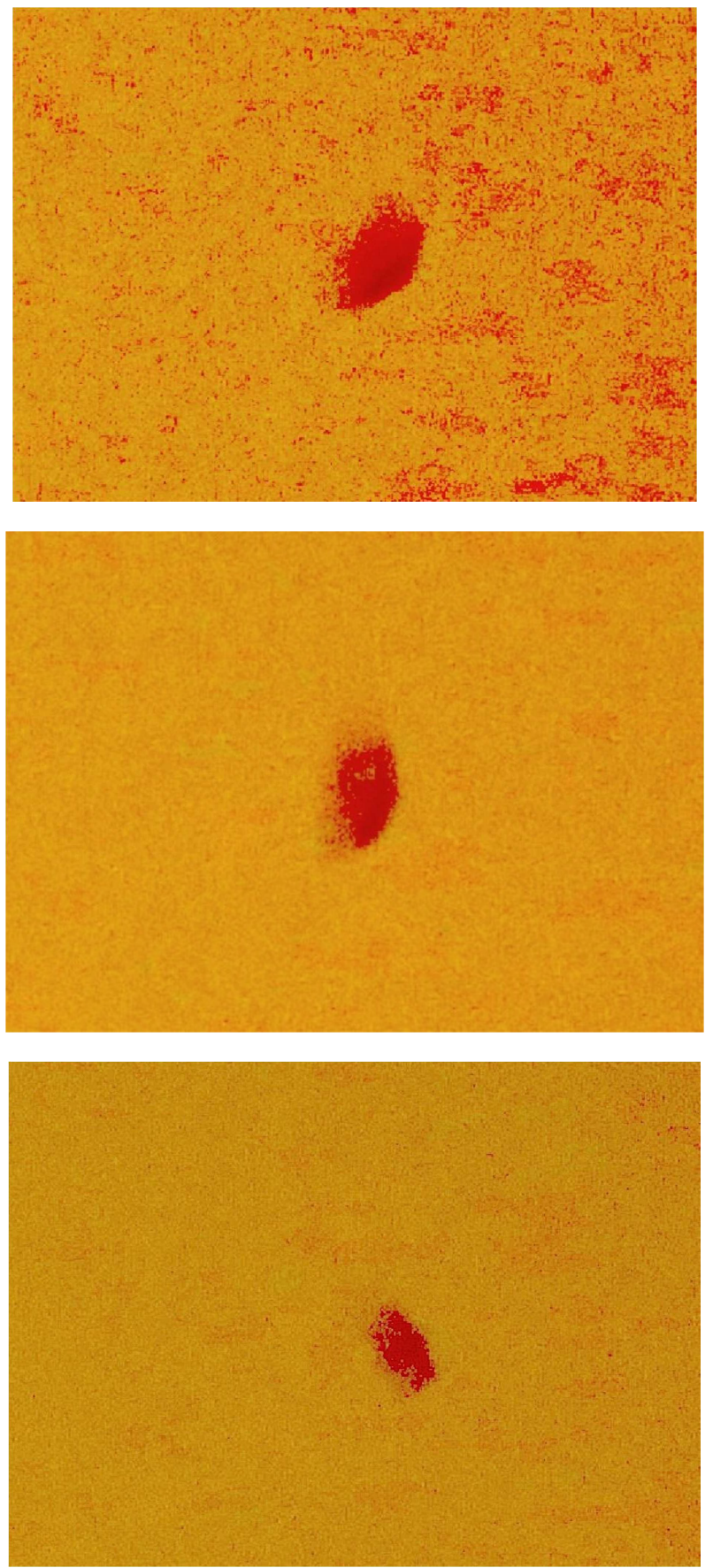

Figure 5. Views of another ITE-1 taken in three consecutive pictures by the digital camera of the Santilli telescope on September 5, 2015 in the night sky over Tampa Bay, Florida. Note that the entity not only moves slowly from photo to photo, but also rotates. This particular type of motion excludes impurities in the telescope lens or in the camera as possible interpretations.
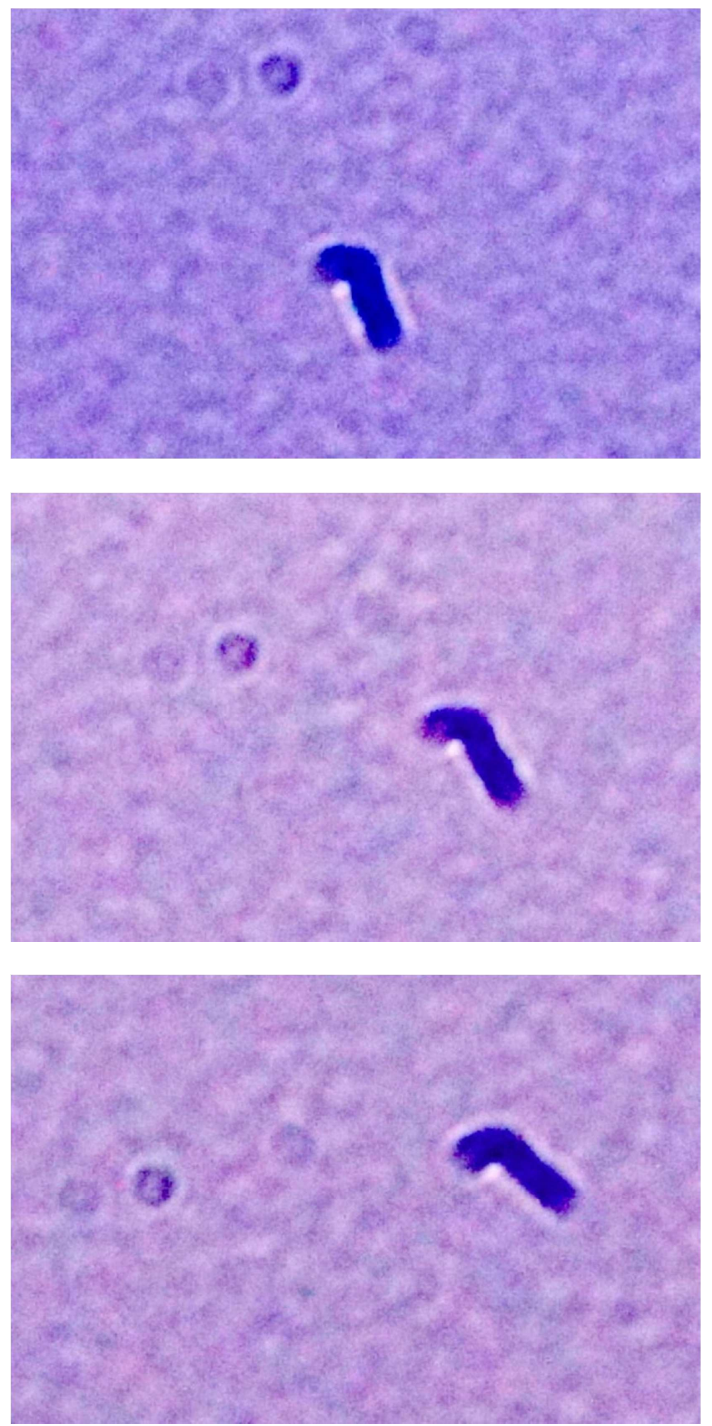

Figure 6. Views of an ITE-1 independently taken by K. Brinkman from the night sky of St. Petersburg, Florida, on September 20, 2015 via the use of the pair of 150 Galileo and Santilli telescopes, under a series of three burst (rapid) shots [13]. The entity is clearly an ITE-1 because it was in our terrestrial environment, it was only visible in the Santilli telescope, and it produced a dark image on the background of the digital camera. Note that this entity too, not only moves with respect to an image caused by an impurity in the telescope lens, but also rotates (see the original report [13] for more pictures and details).

\section{Concluding Remarks}

In works [1-7], the author presented evidence on the apparent existence of antimatter galaxies, antimatter asteroids, and antimatter cosmic rays obtained via dark images in the background of a digital camera attached to a telescope with convex lenses, known as the Santilli telescope.

The same astrophysical entities are completely invisible to our naked eyes as well as to conventional Galileo telescopes with convex lenses because matter-antimatter annihilation mandates that all characteristics of antimatter must be opposite those of matter. Consequently, light emitted by antimatter has an index of refraction opposite that of matter, thus requiring a concave lens for its focusing, and carries 
negative energy as predicted in 1928 by P. A. M. Dirac, thus explaining the dark character of the images.
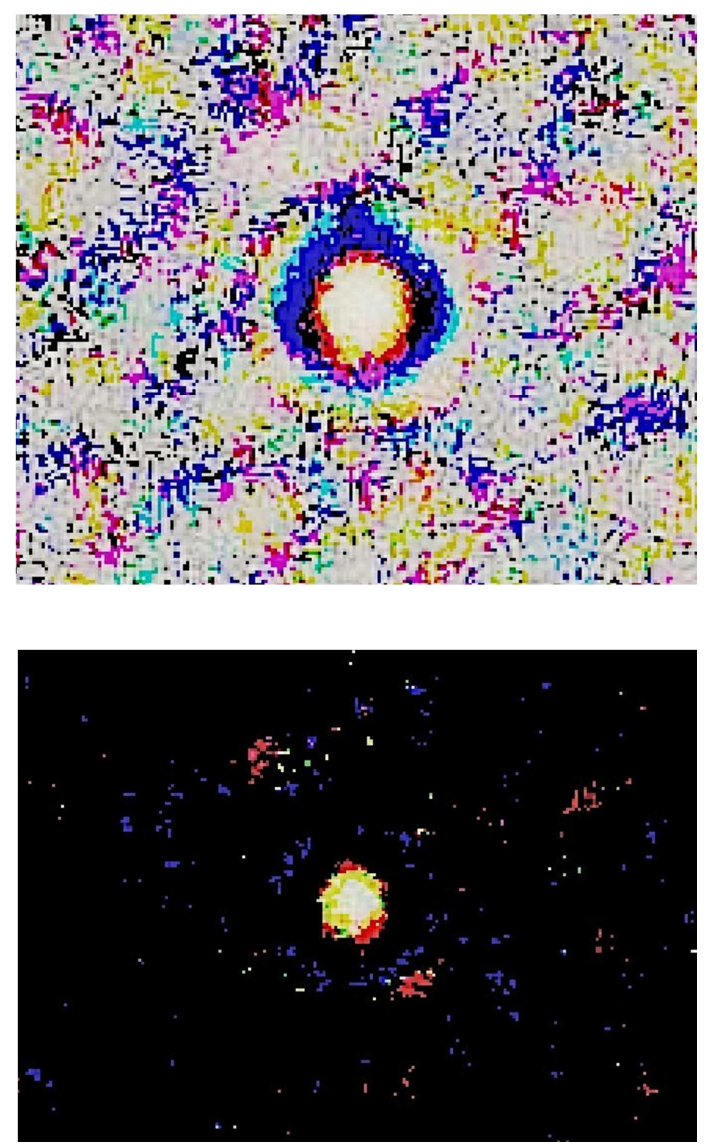

Figure 7. A view in the top of an Invisible Terrestrial Entity of the second kind (ITE-2) taken on September 5, 2015, in the night sky of the Tampa Bay, Florida, from room 775 of the Vinoy Hotel in St. Petersburg at 9.15 pm via the $100 \mathrm{~mm}$ Santilli telescope. We have an ITE-2 because the entity is in our terrestrial environment; it is only visible in the Santilli telescope and it produces a bright image in the digital background, as established by the fact that ordinary light remains visible under strong contrast (bottom view) These data imply that the entity produces light with a negative index of refraction, but with positive energy, thus constituting discovery of a basically new form of light here presented apparently for the first time. In the author's opinion, ITE-2 are indications of structures composed by ordinary matter which emit ordinary light, but achieve invisibility to the human eye as well as to conventional refractive telescopes via the inversion of its index of refraction. This paper has been motivated by the need for our monitoring the possible presence of ITE-1 and/or ITE-2 over sensitive civilian, industrial and military installations since they could be conducting unauthorized surveillance. 11

The historical inconsistencies of negative energies have been resolved for antimatter by the underlying new mathematics specifically constructed for antimatter, known as isodual mathematics, with ensuing, novel, isodual theory of antimatter [1-5].

The above results have been confirmed by a number of independent contributions, such as those of Refs. [8-11]. Ref. [12] provides a comprehensive list of scientific papers published in refereed journals and links to PRWeb Releases in antimatter up to early 2015 .
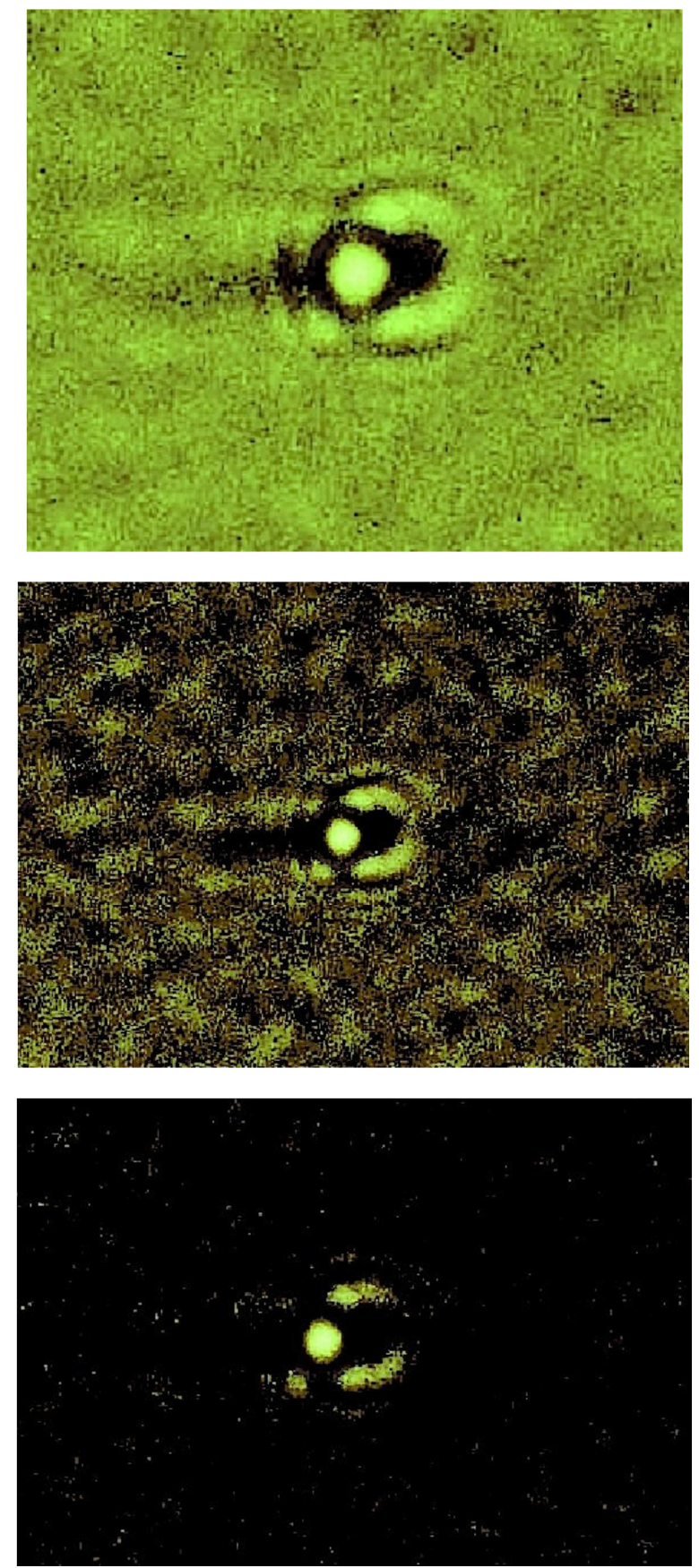

Figure 8. Another ITE-2 (top view) detected under the same conditions as those of the preceding figure. The entity was also invisible to the naked eyes and to the Galileo telescope but fully detected via the Santilli telescope in the night sky of the Tampa Bay. Again, the entity was releasing light with negative index of refraction, yet carrying positive energy as established by the bright residue following maximal contrast (bottom view). Note that this particular ITE-2 moves and, most importantly, causes rings of ordinary light in its surroundings, which can only be quantitatively explained via a gravitational repulsion of ordinary light by this particular entity. On technical grounds, we should recall that the conventional (positive) index of refraction, and the consequential reduction of the speed of light within transparent media, are due to the attraction of light by matter via complex electromagnetic interactions at atomic distances. The negative index of refraction of antimatter-light when passing through a matter-medium such as water, is then expected to be due to the repulsion of antimatter-light by matter. Note finally that the reversal of the index of refraction implies that antimatter light travels in ordinary water at speeds faster than that of light in vacuum [15]. 

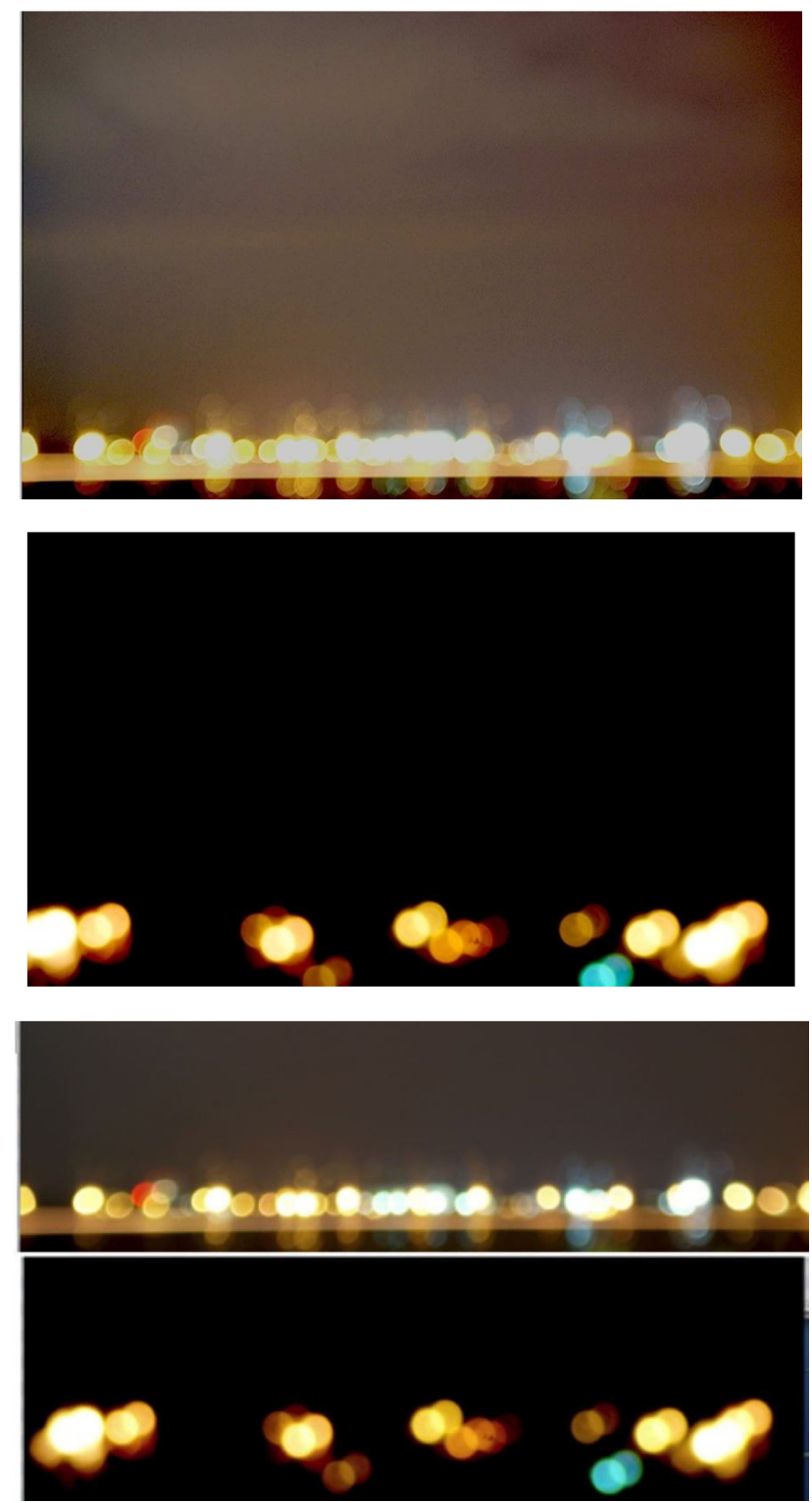

Figure 9. Photos of a cloudy night sky over Tampa Bay, Florida, taken from the third floor of the valet parking garage of the Westin Hotel on September 19, 2015, at 10.20 pm via two Sony cameras SLT-A58K set at ISO Automatic and 15 seconds exposure, attached at the proper focal distance to the pair of $100 \mathrm{~mm}$ Galileo and Santilli telescopes. All photos were taken with simultaneous remote shutters. The photos were taken in the presence of the author, his wife Carla Santilli, and an independent witness. The first photo depicts the lights of the Gandy Bridge in the Tampa Bay as seen from the $100 \mathrm{~mm}$ Galileo telescope without any enlargement. The second photo clearly depicts a bright ITE-2 taken with the $100 \mathrm{~mm}$ Santilli telescope also without any enlargement, which entity was completely invisible in the Galileo telescope as well as to the eyes of the three eyewitnesses. The third picture compares the first photo (top) to the second (below). In comparing the two photos, one should note: 1) The illumination of the clouds in the first photo, and the absence of such an illumination in the second photo; 2) The lights viewed with the Galileo telescope are stationery (except for small fluctuations due to wind), while the lights viewed via the Santilli telescope are pulsating; and 3) Close inspection of the view from the Santilli telescope shows that we are dealing with one single light that, not only pulsates, but moves backward and forward with respect to the telescope in a horizontal plane. This peculiar behavior clearly suggests that this ISE-2 was conducting unauthorized surveillance of the Tampa Area solely visible with the Santilli telescope, thus confirming the need for systematic views of sensitive civilian, industrial and military installations.
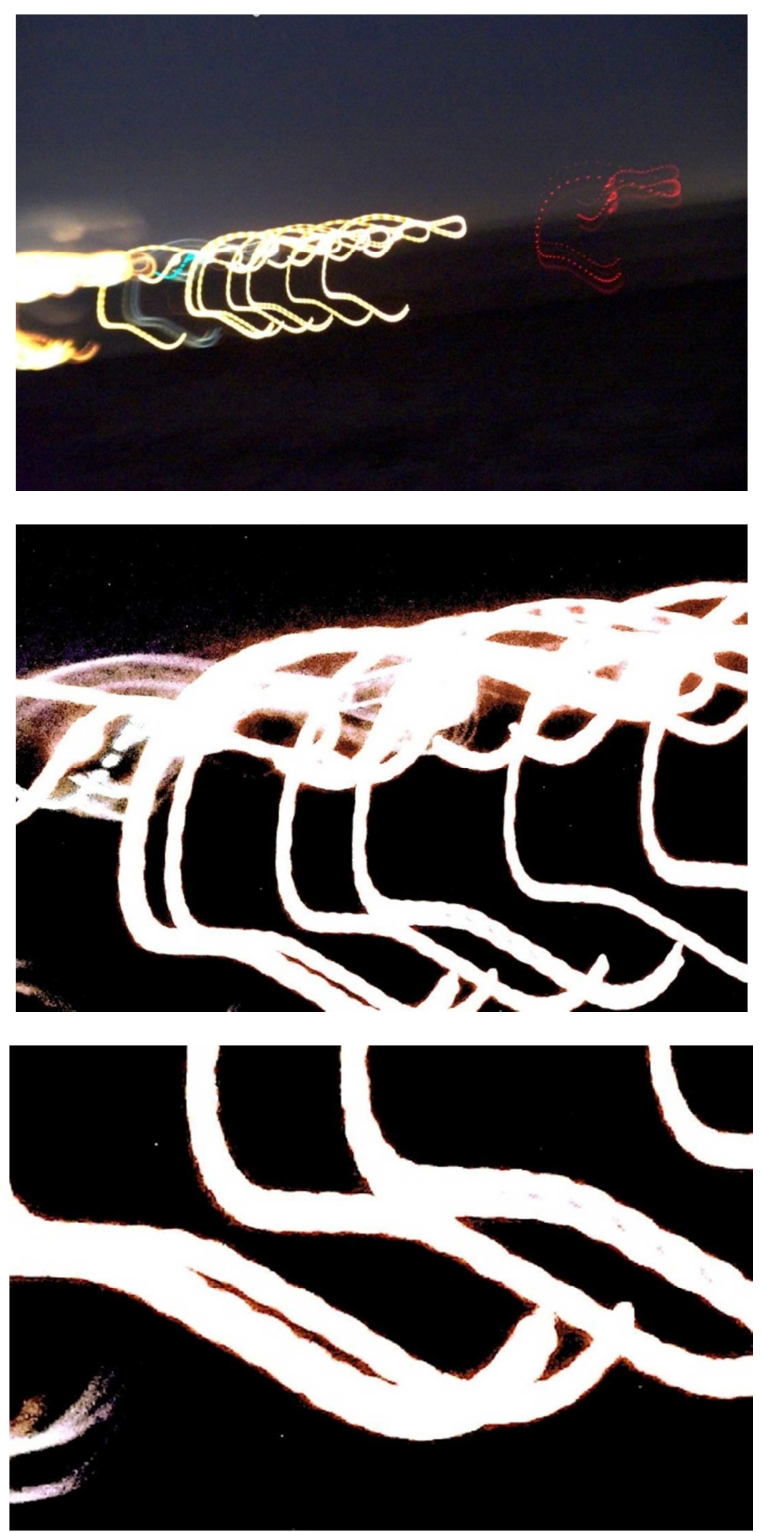

Figure 10. We present here a photo taken on a cloudy night sky of Tampa, Florida, taken from room 775 of the Vinoy Renaissance Hotel, St. Petersburg, on September 5, 2015, at 9:15 pm via two Sony cameras SLTA58K set at ISO Automatic and 15 seconds exposure and connected at the proper focal distance to the pair of $100 \mathrm{~mm}$ Galileo and Santilli telescopes. All photos were also taken with simultaneous remote shutters. No view from the Galileo telescope is here reported due to lack of any meaningful image. The top view depicts a very unusual and bright ITE-2 as seen from the Santilli telescope without any enlargement. The remaining views depict the same photo under progressively increasing enlargements. A first striking aspect is that this particular entity was not seen at all by the author with his naked eyes despite its size and brilliance. A second striking aspect is the complexity of the entity that, due to the 15 seconds exposure, show some clear ongoing operation. The most striking aspect in the view of the author (a theoretical physicist) is the sharpness of the light at its edges, as shown by the last view, since such a feature is against known physical laws requiring light to experience diffraction at its edges with a short yet progressive decrease from full brightness to full darkness. Close inspection of the photo indicates that the entity is in the process of releasing "seven" smaller equally bright ITE also of the second kind, that are reminiscent of the bright ITE-2 of the preceding figure. The unusual features of this particular ITE-2 confirm the need to conduct a systematic surveillance of our sensitive civilian, industrial and military installations, which mandated the writing and release of this paper. 

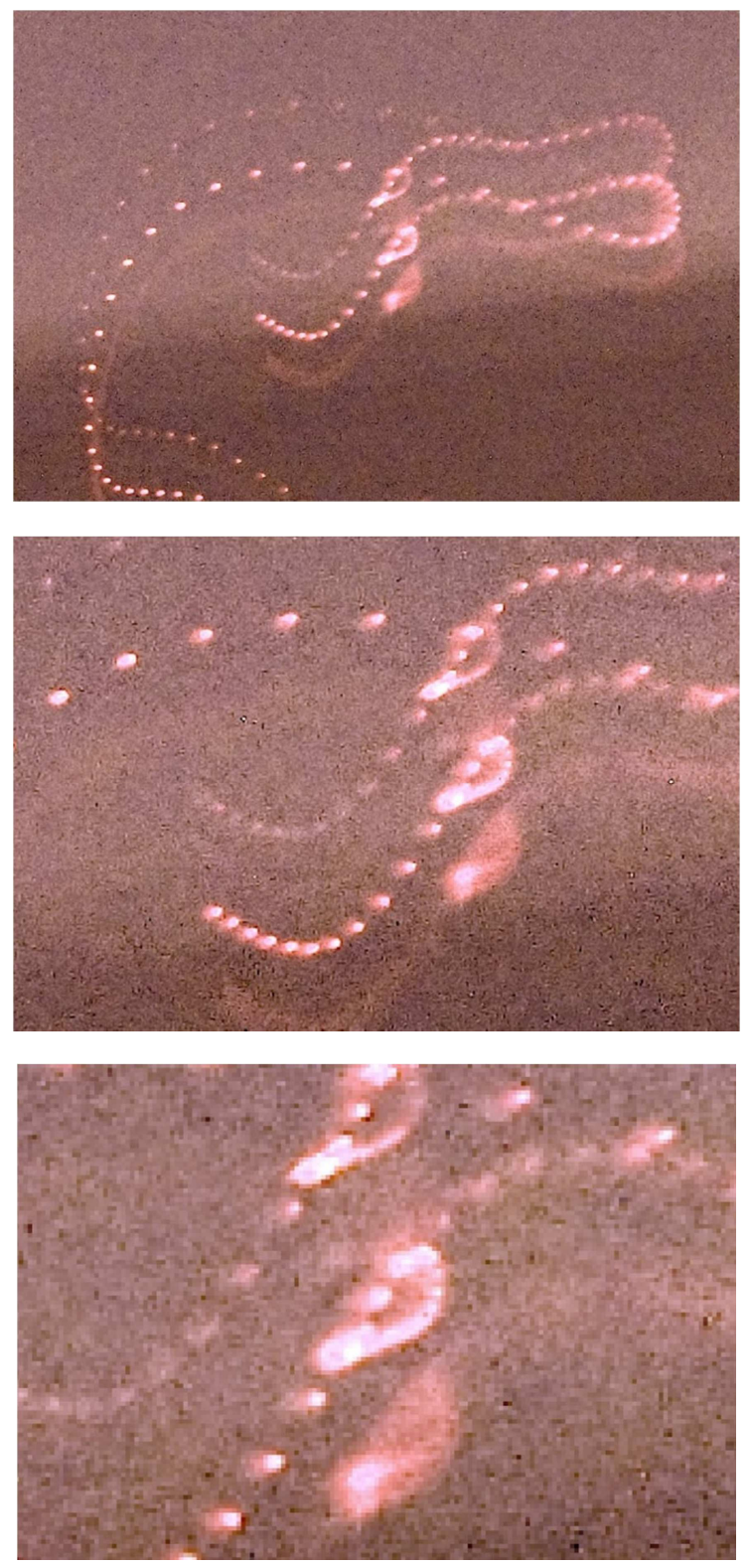

Figure 11. In the top view of this figure we present an enlargement of a second ITE-2 depicted in the top right of the main photo of Figure 10, while the remaining views show progressively increasing enlargements. As one can see, the entity consists of "three" lights of different colors that are pulsating and move synchronously in the cloudy night sky over the Tampa Bay, according to a rather complex trajectory during the 15 seconds exposure, including a point in which motion apparently stopped for a few seconds, to resume thereafter. The synchronous motion of the three separate trajectories strongly suggest that this particular entity consists of one single structure made up of matter that achieves invisibility to the human eyes and to our optical instruments via the creation of a basically new light with negative index of refraction but positive energy presented in this paper apparently for the first time. In the author's opinion, such a behavior suggests again the conduction of unauthorized surveillance of Tampa Bay that motivated the author to write and release this paper.

Via the use of pairs of Galileo and Santilli telescopes, in this paper we have presented for the first time experimental evidence on the apparent existence of two types of entities existing in our terrestrial environment (as defined in Section 1), here called Invisible Terrestrial Entities of the first and second kind (ITE 1 and ITE-2), that are also completely invisible to the naked eyes as well as to Galileo telescopes, yet they are fully visible via Santilli telescopes.

Consequently, both ITE-1 and ITE-2 have a negative index of refraction when propagating in a matter medium such as glass. Their main difference is that the former entities, also called dark ITE, carry negative energy, thus causing dark images in the background of a camera attached to Santilli telescopes, while latter entities, also called bright ITE, carry positive energy, thus causing bright images despite their negative index of refraction.

The detection of ITE-1 and ITE-2 has been independently verified by colleagues [13], and was disclosed for the first time at an invited lecture delivered at the St. Petersburg Astronomy Club on September 25, 2015 [14].

We point out that ITE-1 and ITE-2 must be made up of matter to prevent a cataclysmic explosion due to their immersion in our matter-atmosphere, but their locomotion appears to be based on the internal presence of antimatter and the use of matter-antimatter repulsion, according to a structure studied at the mathematical level in monograph [5].

The above and other aspects imply that ITE-1 and ITE-2 are structures dramatically beyond 20th century knowledge for a number of reasons, such as the complete inapplicability of 20th century knowledge to the classical treatment of neutral antimatter, the change of the index of refraction, the apparent new typo of locomotion, and other reasons [15].

We also point out that the above terrestrial entities achieve invisibility to our eyes and to Galileo telescopes either via the emission of antimatter light as a form of exhaust (ITE-1), or via engineering means capable of inverting the index of refraction of ordinary light (ITE-2).

The writing of this paper has been motivated by the fact that ITE-1 and ITE-2 behave in a manner strongly suggesting the conduction of unauthorized surveillance of our sensitive, civilian, industrial and military installations, thus warranting their societal knowledge.

On scientific grounds, the documentation presented in this paper establishes beyond credible doubt the existence of two new types of light that are totally outside any possibility of treatment via 20th century physical knowledge, but are fully treatable via the new methods [1-15] specifically developed to treat neutral or charged antimatter at the classical as well as particle levels.

On industrial grounds, the findings presented in this paper establish, also beyond credible doubt, the utility of Santilli telescopes, not only for basically novel astrophysical advances, but also for issues pertaining to personal, industrial and national security.

The author would like to stress that, being a scientist, his duty is only that of reporting the documentation on the existence of ITE-1 and ITE-2, with the understanding that their identification, or lack thereof, belongs to the U.S. Government.

The original photos presented in this paper shall be made available to qualified colleagues for supervised inspection. More importantly, pairs of Galileo and Santilli telescopes are in production and subsequent sale in various sizes (Figure 3) 
at the U. S. publicly traded company Thunder Energies Corporation (www.thunder-energies.con), and they can be made available at no cost for supervised verifications anywhere in the U. S. A.

\section{References}

[1] R. M. Santilli, "Isonumbers and Genonumbers of Dimensions 1, 2, 4, 8, their Isoduals and Pseudoduals, and; Hidden Numbers; of Dimension 3, 5, 6, 7," Algebras, Groups and Geometries Vol. 10, 273 (1993), http://www.santillifoundation.org/docs/Santilli-34.pdf.

[2] R. M. Santilli, "Representation of antiparticles via isodual numbers, spaces and geometries," Comm. Theor. Phys. vol. 3, 1994, $\mathrm{pp}$. http://www.santillifoundation.org/docs/Santilli-112.pdf.

[3] R. M. Santilli, "Nonlocal-Integral Isotopies of Differential Calculus, Mechanics and Geometries," in Isotopies of Contemporary Mathematical Structures," P. Rendiconti Circolo Matematico Palermo, Suppl. Vol. 42, 7-82 (1996), http://www.santilli-foundation.org/docs/Santilli-37.pdf.

[4] R. M. Santilli, “Does antimatter emit a new light?" Invited paper for the proceedings of the International Conference on Antimatter, held in Sepino, Italy, on May 1996, Hyperfine Interactions vol. 109, 1997, pp. 63-81, http://www.santillifoundation.org/docs/Santilli-28.pdf

[5] R. M. Santilli, Isodual Theory of Antimatter with Applications to Antigravity, Grand Unifications and Cosmology, Springer (2006), http://www.santilli-foundation.org/docs/santilli-79.pdf.

[6] R. M. Santilli, "The Mystery of Detecting Antimatter Asteroids, Stars and Galaxies," The American Institute of Physics, in press, 2012, http://www.santillifoundation.org/docs/antimatter-asteroids.pdf.

[7] R. M. Santilli, "Apparent detection of antimatter galaxies via a telescope with convex lenses," Clifford Analysis, Clifford Algebras and their Applications vol. 3, 2014, pages 1-26 (Cambridge,UK),http://www.santillifoundation.org/docs/Antimatter-telescope-2013-final.pdf.
[8] P. Bhujbal, J. V. Kadeisvili, A. Nas, S Randall, and T. R. Shelke, "Preliminary confirmation of antimatter detection via Santilli telescope with concave lenses," Clifford Analysis, Clifford Algebras and their Applications Vol. 3, pages 27-39, 2014 (Cambridge, UK). http://www.santillifoundation.org/docs/Con-Ant-Tel-2013.pdf.

[9] S. Beghella-Bartoli, P. M. Bhujbal, Alex Nas, "Confirmation of antimatter detection via Santilli telescope with concave lenses," American Journal of Modern Physics Vol. 4, pages 34-41 (2015), http://www.santillifoundation.org/docs/antimatter-detect-2014.pdf.

[10] R. Anderson, A. A. Bhalekar, B. Davvaz, P. Muktibodh, V. M. Tangde, and T. Vougiouklis, "An introduction to Santilli's isodual representation of antimatter and the ensuing problem of detecting antimatter asteroids," Numta Bulletin Issue 67, pages 1-33, $2013 \quad$ http://www.santillifoundation.org/docs/Antimatter-2013.pdf.

[11] S. Beghella Bartoli, "Trajectories of antimatter asteroids in our solar system according to the isodual theory of antimatter," Hadronic Journal Vol. 37, pages 1-27, 2014 http://www.santilli-foundation.org/docs/Simone-FINAL.pdf.

[12] P. Fleming, "Scientific references and PR Web News Releases on Santilli isodual Theory of Antimatter, http://www.santillifoundation.org/docs/Santilli-Telescope-Refs-1-15.pdf.

[13] K. Brinkman, "Santilli Refractors," power point from a lecture delivered at the St., Petersburg Astronomy Club on September $25, \quad 2015$ http://www.thunder-energies.com/docs/SantilliRefractor.pdf.

[14] R. M. Santilli, "The Mystery of Antimatter," Lecture delivered on September 25, 2015 at the St. Petersburg Astronomy Club, http://www.world-lecture-series.org/level-ix.

[15] R. M. Santilli, "Compatibility of Arbitrary Speeds with Special Relativity Axioms for Interior Dynamical Problems," American Journal of Modern Physics, in press (2016) http://www.santilli-foundation.org/docs/superluminal-speeds. 